\title{
Outbreak Management Guideline of Chikungunya Fever: Bangladesh Perspective
}

ASM Alamgir
Chikungunya Fever is a viral infection and it is caused by the RNA virus named chikungunya virus. It is an arbovirus (Arthropod Born Virus). This virus is transmitted by Aedes mosquito. It is very interesting that Aedes mosquito is more prevalent in Bangladesh. There are many safe breeding places for this mosquito. Therefore, this virus can easily infect at any age group of the community. In these context development guidelines is an important issue.

Entomological examination has proved that Aedes aegypti is the common vector responsible for transmission in urban areas whereas Aedes albopictus has been implicated in rural areas. It has been established that the Aedes mosquito breeds in domestic setting such as flower vases with clean water, water storage containers air coolers water reservoir, construction sites, coconut shells, containing clean water, tures, plastic, metal cans which is stored more than three days.

In the South-East Asia region, chikungunya virus persists in the human population by human mosquito human transmission cycle. During post monsoon season a high vector density leads to increase the transmission Communicable Disease control (CDC) of Directional General of Health services under Ministry of Health \& Family welfare of Bangladesh with the collaboration of IEDCR has planned to develop the management guidelines for chikungunya fever. These guidelines will give the proper diagnosis \& treatment in IEDCR, the chikungunya a virus is confirmed by IEDCR by RTPCR. Furthermore the ICT devices are also available to detect the chikungunya virus specific antibody which is both IgG and IgM. However it is very interesting that WHO approved ICT kit has poor detection capacity which is from my personal experience of view. The RT-PCR confirmed cases have shown ICT negative for IgG and IgM. This is a very confusing result.

There is a dilemma of clinical diagnosis of chikungunya fever. The reason is that clinical features of chikungunya fever have the similarities with Dengue fever leads to diagnostic confusion and management of the cases. The main cardinal features of chikungunya fever are high fever, rash, body ache with arthritis or joint manifestation. Vomiting is also an important symptom of chikungunya fever. This leads to electrolyte imbalance. However, bleeding manifestation is one of the important complication of Dengue fever which is absent in chikungunya fever. There is only symptomatic treatment. There is no current specific treatment available for chikungunya fever. Like Dengue fever, fluid management is essential tools for primary management with application of paracetamol. This medicine will cover both joint pain as well as high fever. NSAIDS can be given with special precaution.

All these management is maintained in the guidelines. Thus this guideline will give a management tools to the primary to tertiary care hospital. According to the guidelines the physicians and Sub-Assistant Community Medical Officer (SACMO, previously known as medical assistant) will be trained up in the Institute of Epidemiology, 
Disease Control \& Research, Dhaka (IEDCR) so that they can manage the case easily whenever there is an outbreak occurs in this country.
Bangladesh Journal of Infectious Diseases 2017;4(1):1-2

How to cite this article: Alamgir ASM. Outbreak Management Guideline of Chikungunya Fever: Bangladesh Perspective. Bangladesh J Infect Dis 2017;4(1):1-2 